\title{
Formalizing mobility in dynamic location-aware sensor networks
}

\author{
Michael Worboys* and Matt Duckham ${ }^{\dagger}$
}

\section{Introduction}

This is a short position paper that outlines work on the development of a formal model of mobility and process in location-aware sensor networks. Formal models are important when there is a need to verify or reason about properties of the system and its environment. In ontological terms, we can make a distinction between "continuant" and "occurrent" entities in the sensor network [2]. Continuants are the enduring entities in the domain, such as sensors and physical objects in the sensed environment, along with their attributes and inter-relationships. Occurrents are the events, activities, and changes in the network and its environment. Examples of occurrents are sensor movements, changes in network linkages and properties, events in the sensed environment, as well as protocols that constrain these actions. In our view, the dominant features of a dynamic network are its occurrent entities, and this is the focus of our work. Formal models for representing and reasoning about occurrents include the event calculus [3] and process calculi of Milner [4, 5]. More recent work by Milner in the development of the $\pi$ process calculus [6] allows the possibility of formally modeling mobile processes, and this is the line of development followed here. Our goal is to show that process calculi can provide an integrated formal model of location-aware sensor networks and their environments that complements the simulation approach (e.g., ns2, GloMoSim, TOSSIM, and Ptolemy/Visual Sense).

\section{Formalizing mobility in processes}

The general application of Milner's process calculus to geospatial occurrents is discussed in [7], while early work on formal models of dynamic spatial fields as sensed by geosensor networks is reported in [1]. In this section, we introduce the basic elements and combinators for representing processes. Single processes may be viewed as compositions of atomic actions. An action is an indivisible,

\footnotetext{
* National Center for Geographic Information and Analysis, University of Maine, Orono ME 04469, USA

${ }^{\dagger}$ Department of Geomatics, University of Melbourne, Parkville, Victoria, 3010, Australia
} 
occurrent entity. Actions, like the processes of which they form part, are situated in space-time. The basic way for actions to combine is sequentially. If $P$ and $Q$ are processes, and if $Q$ is constructed as action $a$ followed by process $P$, we may write this as an equation to define $Q$ :

$$
Q \stackrel{\text { def }}{=} a . P
$$

Another basic concept in process construction is disjunction, represented by the summation operator + . For processes $P, Q, R$, and actions $a, b$, the definitional equation:

$$
R \stackrel{\text { def }}{=} a \cdot P+b \cdot Q
$$

shows that $R$ is the name for the process that allows either the action $a$ followed by process $P$ or $b$ followed by $Q$.

The key ideas in multi-process interaction are concurrency and synchronization. Processes are $P$ and $Q$ are concurrent if they run together, not necessarily with any interaction. The process calculus operation is concurrent composition, and written $P \mid Q$, for processes $P$ and $Q$. Synchronization is the operation whereby processes can interact. A synchronization can occur between two concurrent processes, when one process has an output action ready to run, and the other a complementary input action. Actions can take variables by which messages are passed at a synchronization. Each pair of complementary actions constitutes a channel of communication between the two processes. For example, the parameterized output action $\overline{a(v)}$ has the capability of passing the data $v$, which another process with input action $a(x)$ may receive by substituting $v$ for $x$ in free occurrences of variable $x$. For two concurrent processes $P(v) \stackrel{\text { def }}{=} \overline{a(v)} \cdot P^{\prime}$ and $Q(x) \stackrel{\text { def }}{=} a(x) \cdot Q^{\prime}(x)$ there is a possibility of a transition, written $P(v)\left|Q(x) \rightarrow P^{\prime}\right| Q^{\prime}(v)$. Because the $\pi$-calculus allows synchronizing concurrent processes to communicate not only actions but also channel names, the framework provides the capability to realign channels of communication and hence to model mobility.

\section{Process calculus applied to mobility in location- aware sensors}

In this section we demonstrate the application of process calculus constructs to the development of formal models of mobility in location-aware sensor networks. Two kinds of mobility are considered: changes in the sensor communication network, and physical movement of sensors. In this short paper the explanations omit formal details, and we do not model changes to the sensor environment. Where diagrams are used for explanation, processes are represented by labeled boxes, their ports indicate input and output actions, and channels of communication represented by lines connecting complementary action pairs.

As an initial approximation, a sensor network can be modeled as a collection of mobile concurrent processes (sensors), and interactions between processes through channels (communication between sensors). Figure 1 illustrates 


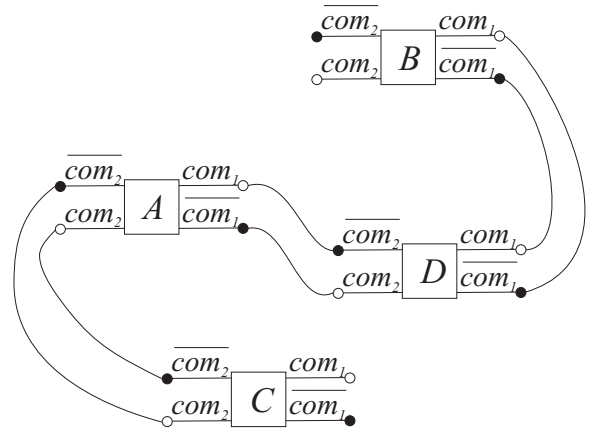

a

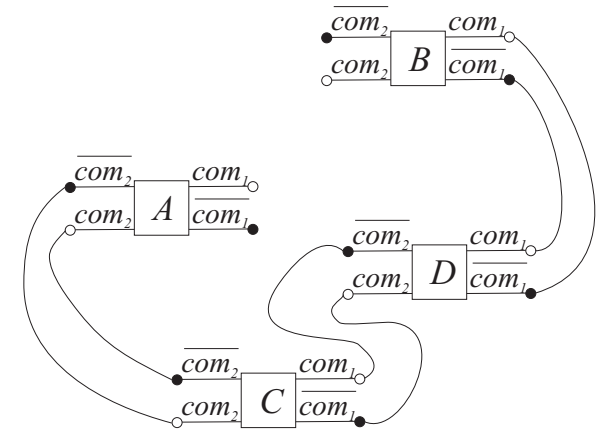

b

Figure 1: Network mobility in sensor networks: sensor $D$ moves communication channel from $A$ to $C$

the process-oriented model of a small sensor network, where in this case edges represent capability for one-hop communication between sensors. The communication channels may also be parameterized, modeling message passing between sensors. Using $\pi$-calculus we can model one type of mobility in sensor networks as the realignment of communication channels. For example, in moving from Figure 1a to Figure 1b, one channel of communication (com) for sensor $D$ has moved, perhaps modeling sensor link failure and reactivation. The protocols for realigning channels and passing messages (not shown in the diagrams) can also be formally encoded in the process calculus.

A second type of mobility concerns the physical movement of sensors to new locations. A key advantage of adopting process calculi as a formal model of sensor networks is that the same basic formal components can be reused to model different types of mobility. For example, Figure 2 shows geographic locations represented as processes (circular boxes). In this case, adj-channels between locations model physical adjacency and occ-channels between locations and sensors represent occupancy (i.e., a sensor occupying a location). Physical sensor mobility can then be represented as the realignment of occupancy channels. The process calculus protocols for channel realignment can be designed to encode the physical constraints of the systems (e.g., sensors must move continuously, transitioning only between adjacent locations and not jumping directly between disconnected locations) and may or may not be independent of other channel realignments (e.g., realignment of communication channels may depend on physical location, with only sensors located near one another being able to communicate).

Although representing geographic locations as processes is initially counterintuitive, it does have the advantages of conceptual, formal, and potentially computational efficiency (it is an explicitly qualitative, discrete model of space). Figure 3 shows two possible realizations of such a model, where processes may represent geographic regions in 2D space or nodes in a graph in network space. 


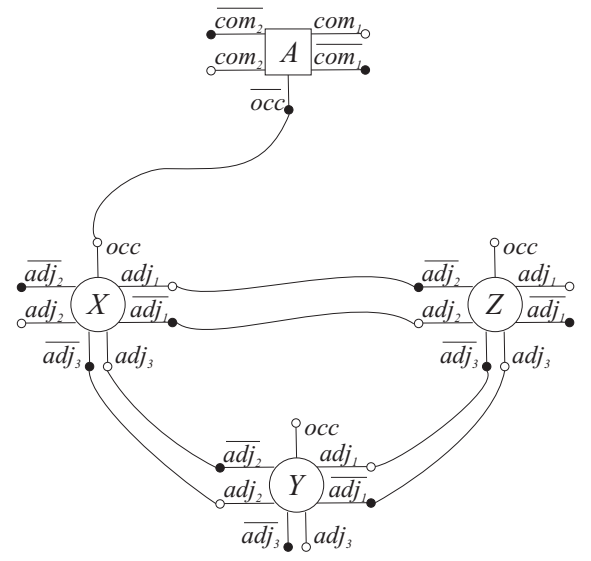

a

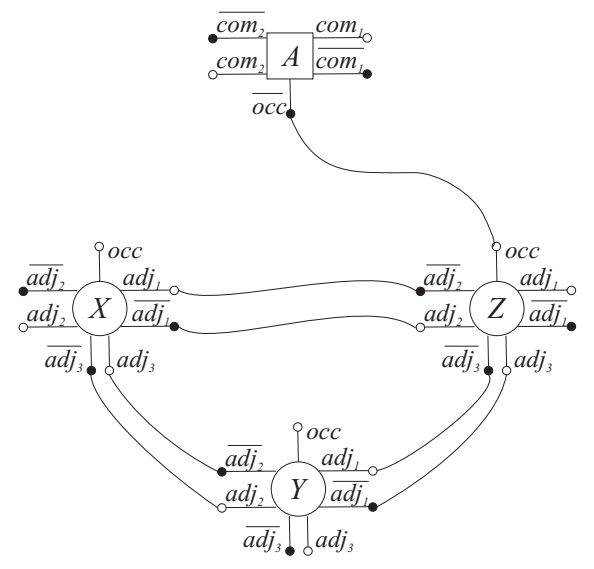

b

Figure 2: Geographic mobility in sensor networks: sensor $A$ moves from $X$ to Z

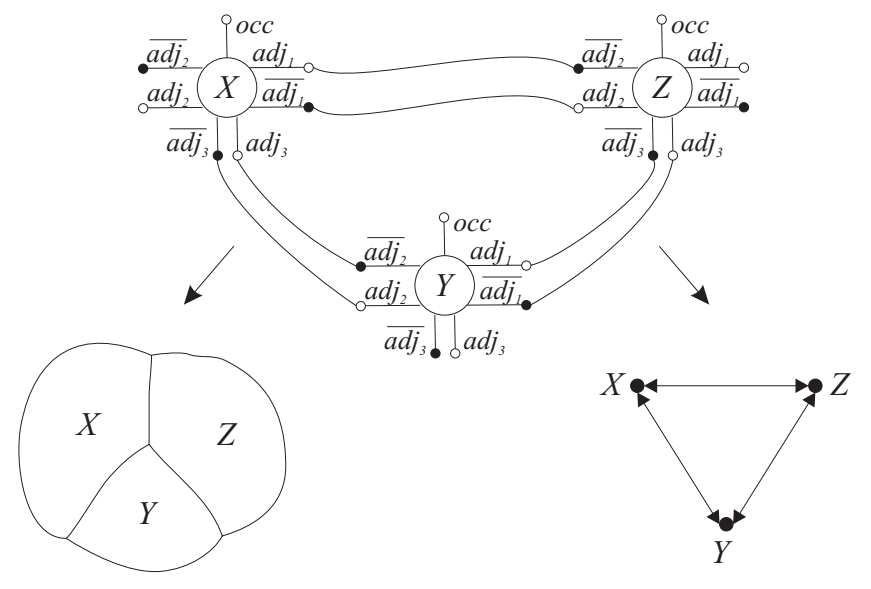

Figure 3: Two geographic interpretations of locations as processes: regions in 2D plane and nodes in a network 


\section{Summary}

Our premise is that formal specification of sensor networks and network protocols is an important complement to empirical modeling techniques currently used, and that process calculi provide a suitable formal framework. Formal models are important when there is a need to verify or reason about properties of the system and its environment. The advantages of the formal model provided here are that it provides, within a small number of formal constructs, a unified framework for modeling sensor network and environment, and that it has the power to formalize mobility in both settings.

Current work is the construction of a demonstrator, in collaboration with the Ordnance Survey of Great Britain, in the case of a location-aware network in the transportation domain. Key aspects of this work are construction of the formal model and development of a database design and implementation based on this model.

\subsection{Acknowledgments}

Matt Duckham is partially supported by the Australian Research Council under ARC Discovery Grant DP0662906. Mike Worboys work is supported by the National Science Foundation under NSF grant numbers IIS-0429644 and BCS-0327615. Both are supported by the Ordnance Survey of Great Britain.

\section{References}

[1] Matt Duckham, Silvia Nittel, and Mike Worboys. Monitoring dynamic spatial fields using responsive geosensor networks. In GIS '05: Proc. 13th annual ACM international workshop on geographic information systems, pages 51-60, New York, NY, 2005. ACM Press.

[2] P. Grenon and B. Smith. SNAP and SPAN: Towards dynamic spatial ontology. Spatial Cognition and Computation, 4(1):69-104, 2004.

[3] R.A. Kowalski and M.J. Sergot. A logic-based calculus of events. New Generation Computing, 4:67-95, 1986.

[4] R. Milner. A Calculus of Communicating Systems, volume 92 of Lecture Notes in Computer Science. Springer Verlag, 1980.

[5] R. Milner. Elements of interaction(Turing Award Lecture). Comm. ACM, 36:78-89, 1993.

[6] R. Milner. Communicating and Mobile Systems: The $\pi$-calculus. Cambridge University Press, 1999.

[7] M.F. Worboys. Event-oriented approaches to geographic phenomena. International Journal of Geographic Information Science, 19(1):1-28, 2005. 